monastery in Jerusalem, and there may have been no longer an abbot; possibly 'prior, canons, and brethren of the Temple' was a mere formula, and there was then no house of which Ferriby was a cell. ${ }^{22}$ Nothing seems to be known of the convent of the Temple after the fall of Jerusalem; and the very existence of the 'order of the Temple' was apparently unknown to Pennotti, a canon regular of the Lateran congregation, the historian of his order.

Egerton Beck.

\title{
Confirmations of Oxford Chancellors in the Lincoln Episcopal Registers
}

THE subject of dispute between the university of Oxford and the bishops of Lincoln concerning the chancellorship, ${ }^{1}$ of which records, extending over nearly a century, exist in the Lincoln Registers, was whether the chancellor elected by the university was bound to go in person to the bishop to receive his confirmation, or whether he might receive it by proxy. The bishops asserted the former, the university the latter. In the Lincoln Episcopal Registers there are twenty-nine entries of confirmation, six being among the Memoranda and twenty-three among the Institutions. No transcripts of the latter are found in the collections of Twyne, and so presumably the documents were unknown to Wood. The earliest date at which a commission is entered in the registers is 1290 , when the episcopal records began to be written on quires of parchment instead of membranes stitched together to form a roll. From 1290 the commissions continue almost without a break until the episcopate of Gynwell, whose period of office was marked by the most memorable of all disputes connected with the chancellorship. Having refused persistently to confirm William de Palmorva, the bishop was excommunicated by the archbishop of Canterbury, who himself confirmed the chancellor. ${ }^{2}$ After such a grievous quarrel it is strange to find

\footnotetext{
": A century later Ferriby was certainly an independent house or it would bavo been suppressed as an alien priory.

'Wood's Annals, i. 326, 329, 346, 451, 481 ; Annales Monastici, iv, 317; Rot. Parl. i. 16; Rashdall's Universities, ii. 424, 426. The origin of the dispute is nowhere recorded. The chancellorship was created by the bishop of Lincoln in 1214 (Munimenta Academica, p. 2; Wood, i. 184; Rashdall, ii. 351), and the dispute began at a date not far removed from the inception of the office if we may judge from Bishop Sutton'sstatement, 'quod beatus Robertus quondam Lincolniensis Episcopus, qui buius. modi officium gessit dum in vniversitate predicta regebat, in principio creationis sue in episcopum dixit proximum predecessorem suum episcopum Lincolniensem non permisisse quod idem Robertus vocaretur Cancellarius sed Magister Scolarum' (Sutton's Mem. f. 117; Rashdall, ii. 355). For references to 'Magister scholarum' see Hist. MISS. Comm., Duke of Rutland's MSSS. iv. 82 ; Rashdall, ii. $35 \overline{3}$.

= Wilkins's Concilia, iii, pp. 3-8; SI un. Atad. p. 168
} 
Bishop Gynwell ultimately numbered among the university's benefactors. ${ }^{3}$ Only two commissions, dated 1363 and 1367 respectively, are found in the register of his successor, Buckingham. On 8 Nov. 1367 the university was freed from episcopal jurisdiction by the bull of Urban $V,{ }^{4}$ notwithstanding which the bishop, on 4 May 1369, cited Adam de Toneworth, who had recently become chancellor, to appear before him for having been irregularly elected. $\mathrm{He}$ was again cited in the same year, and in all probability the citations were ignored. ${ }^{5}$

The procedure of confirmation is similar in all the commissions, and reduced to its simplest form is as follows. The university sends messengers to the bishop with letters close requesting the confirmation 'more solito' of their elected chancellor. Letters patent introducing the messengers as the representatives of the university are next presented. Excuses having been offered for the chancellor's absence, the bishop confirms briefly the nominated chancellor in his office 'de gratia speciali ... donec aliud vobis super hoc dederimus in mandatis'. Wetheringsete's commission, which has many points of difference, is by far the most detailed of the records of confirmation found in the registers. ${ }^{6}$

The messengers of the university in 1291 and 1293, the first two recorded occasions when such were sent, were a master of canon law and a master of arts. On the two following occasions a single university representative appeared before Bishop Sutton. By the time a new chancellor had been elected Dalderby had become diocesan, and on the university again sending a single messenger the bishop remonstrated. During the remainder of his episcopate a master of canon law, a master of civil law, and a proctor were usually sent; not always, however, men of authority-at least in the bishop's opinion. He is found inquiring 'quare maiores vniuersitatis ad episcopum non venerunt sicut fieri consueuit ', 7 and to a question put by him to the messengers who sought the confirmation of William de Bosco, the reply was 'ignoramus quia iuvenes sumus et per tempus modicum in vniuersitate reximus '.${ }^{8}$ In October 1322 , the first time messengers were sent to his successor Burghersh, representatives from the five faculties of theology, canon law, civil law, medicine, and arts were chosen. ${ }^{9}$ Two months later statutes were promulgated in the university dealing with the office of chancellor, and

3. $1 /$ uu. . Acad. p. 187 ('Joh. de Synewelle').

- .Mun. Acad. p. 2288; Cal. of Papal Reg., Letlers, iv, pp. 66, 83.

s Buckingham's Mem. ff. 70, 73 .

- I have to thank the Rev. H. E. Salter for a few of the following facts, and MIr. G. W. Wheeler for some suggestions and corrections. In vol. xxir, 735 ante, I omitted to record that my knowledge of the transeript in the Smith MS. was due to the kindness of Mr. F. Madan.

- Ibid. f. 153.

7 Dalderby's Instit. f. 144.

" Burghersh" s Instit. f. 248. 
by these it was provided that when a chancellor was elected ' pro nova commissione habenda, per unum vel duos Magistros ad plus, super huiusmodi negotio ad plenum instructos mittatur '. ${ }^{10}$ Thenceforward a professor of civil law and a proctor were generally the representatives of the university.

The excuses offered for the absence of the chancellor are interesting features of the commissions. The first recorded excuse is that the chancellor was already well known to the bishop, ${ }^{11}$ which was evidently a retort to Sutton's objection to give a commission 'personae sibi incognitae'.12 Expense and interruption of study were frequently put forward. One of the excuses for Cobham's absence was that if he had come in person ' oportuisset secundum morem solitum quod de singulis facultatibus secum venisset unus regens, et sic in eisdem facultatibus cessatum fuisset eis absentibus ad magnum studentium detrimentum ', ${ }^{13}$ and for Wetheringsete's 'si venisset cessatum fuisset in ipsius absencia a lectura per alios magistros professionis sue '.14 Disturbances in the university due to the absence of the chancellor were also urged. In Favresham's commission reference is made to the evil deeds of a faction called Lucan. ${ }^{15}$ Burdon's excuse was that the justiciars of the king were at Oxford, and it would therefore be unseemly for him to leave the town. He had, moreover, a sermon due on Ash Wednesday. ${ }^{16}$ William de Bosco could not appear ' quod non habuit eveccionem paratam pro tanto domino et quod eveccio ipsius fuit in loco multum distanti ab vniuersitate '. ${ }^{17}$ By the time of Bishop Burghersh all the various excuses for absence had crystallized into 'propter pericula quae in absentia Cancellarii inter scolares ad Universitatem Oxon. undique confluentes potuerunt de facili imminere'. In the last commission found in the registers, that granted to William de Courtney in 1367, the messengers made a bolder reply-the chancellors were not accustomed to come in person. The bishop asserted the contrary, and declared that unless they came in person he would not confirm. After some argument the messengers supplicated that the bishop would confirm ' de gratia ista vice', to which he replied, " ex quo petitis graciam contemplacione persone sic electe volumus vobis ista vice graciam facere specialem," cum protestacione quod non intendebat derogare in aliquo iuri suo per huiusmodi graciam eis hac vice concessam, sed protestabat expresse quod noluit de cetero aliquem sic electum admittere nec confirmare nisi veniret in propria persona ${ }^{\prime}{ }^{18}$

10 Mun. Acad. p. 107.

"Sutton's Mem. f. 50".

12 Annales Mfon. iv. 317.

${ }^{23}$ Dalderby's Mem. $5^{\nabla}$ : Twyne ii. f. 4.

14 Ibid. f. 143". The chancellor was invariably chosen from the faculties of theology and canon law : Mun. Acad. p. 493.

16 Dalderby's Instit. f. $145^{\text {\% }}$.

17 Ibid. f. 153.

16 Ibid.f. 149.

28 Buckingham's Instit. f. 342. 
The uniformity of phrase and formula is a very marked feature in all the commissions, especially in the letters presented by the university and the letter of the bishop confirming the commission. The uniformity of university rhetoric was probably largely due to the restraining and conservative tendencies of the diocesan, who, mindful of inconvenient precedents, refused to be disarmed even when it was reported that alterations had been made solely to do him greater honour. ${ }^{19}$ Previous commissions were frequently consulted with a view to ascertaining whether procedure accorded with ancient usage, and the collation was evidently performed in no perfunctory manner..$^{20}$ A deliberate attempt seems to have been made to alter the form of letters when Richard de Clive was confirmed. Gilbert de Segrave, the university's messenger, presented a letter which differed from any preceding it in the registers, and which contained a not very appropriate reference to 'fratribus nostri collegii'. This letter having been collated with previous letters and found different, the bishop censured it; whereupon Gilbert de Segrave 'exhibuit statim aliam litteram sub hac forma', which was the usual one referring picturesquely to those who seek the pearl of knowledge in the field of learning. ${ }^{21}$

Episcopal interference seldom preceded the nomination of a chancellor, but in 1314 Bishop Dalderby addressed a letter to the official of the archdeacon and dean of Christianity commanding him to have proclaimed in every church in Oxford that no one under pain of excommunication 'pro cancellario se gerere uel in aliquo ad officium cancellarie spectancia exercere quouis modo presumat, aut banna seu denunciaciones nomine cancellarii vniuersitatis predicte edi faciat seu proponi, et ne quis pareat cuiquam de facto ad pretactum officium pertinencia exercenti, donec per nos aliquis in ipsius vniuersitatis Cancellarium sit prefectus '.22 This letter is immediately preceded in the register by another in which Dalderby quotes a letter of Sutton directed against those in the university who presume to give effect to statutes calculated to prejudice the rights and liberties of the see. Again, in 1343, on a similar occasion, Bishop Becke commanded the senior professor of theology to warn all those 'qui de ipsius cancellarii prefeccione fuerint tractaturi et in eiusdem nominacione interfuerint, quod per confederacionem seu conspiracionem aut machinaciones illicitas ad predictum officium neminem nominare presumant '. .3

Apparently the only commission ever reroked was that of

"Dalderby's Instit. f. 143 ".

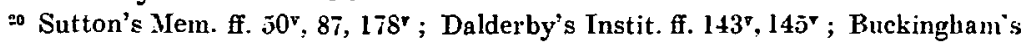
Instit. f. 335 .

= Dalderby's Memn. f. 265.

$\because$ Sutton's Mem. f. $178^{7}$.

s Becke's Mem. f. 33. 
John Lutterel, but the bishop was careful to add in his letter that he had done so with no intention of setting a precedent or of infringing the privileges of the university, but had acted ' vestro rogamine mediante $\cdot .24$

The three following documents represent respectively the first record in detail of a commission, the most elaborate record, and an example of the later conventional form.

\section{Strickland Gibson.}

Sutton, Memoranda, f. $\overline{\mathbf{s}} \mathbf{0}$.

Registrum nominacionis magistri Simonis de Gandauo in Cancellarium Oxon.

Memorandum quod xvj kal. Ianuarii anno domini $\mathrm{M}^{\circ} \mathrm{CC}$ Nonagesimo primo apud Wuburn iuxta Wycumb Episcopo existente in manso Rectoris ibidem in camera mane venerunt ad eum Magister Thomas de Cobeham in decretis et Magister Iohannes de Mora in artibus apud Oxon. actualiter tunc regentes Episcopo exhibentes litteram Universitatis Oxon. clausam sub hac forma.

Reverendo patri in Christo ac domino domino Oliuero dei gracia Lincoln. Episcopo vniuersitatis Oxon. cetus humilis Magistrorum salutem et obedienciam debitam et deuotam. Affeccionis paterne dileccionem habere vos condecet circa querentes in agro studii sciencie margaritam que domum dei multipliciter conuenustat in hiis precipue que non possunt absque denegacione gracie ac iuris iniuria denegari. Hine est quod cum Magister Simon de Gandauo doctor sacre theologie a nostra vniuersitate [f. 50"] ad officium cancellarie per cessionem magistri Iohannis de monemuta vacantis concorditer sit electus, a vestre paternitatis beniuolencia eius eleccionem more solito petimus confirmari. Talem ergo si placet vos exhibeatis in hac parte ut filiorum vestrorum sinceritas augeatur et paterne dileccionis caritas comprobetur. Valeat paternitas uestra reuerenda tempore diuturno.

Qua perlecta et plenius intellecta quesitum fuit a dictis magistris an alia haberent exhibenda pro negocio pro quo uenerunt. Et responderunt quod non. Deinde ipsis secedentibus et Episcopo aliquantulum deliberante cum suis iterum quesitum fuit a magistris predictis utrum haberent aliquod procuratorium nomine vniuersitatis ad prosequendum negocium supradictum, qui dixerunt quod non, adicientes quod non fuerat moris ut credebant. Cuius pretextu examinata fuerunt registra priora de commissionibus prius factis in hoc casu, per que constabat procuratoria pro prosecucione huius negocii prius sepius fuisse transmissa. Fuit insuper quesitum a magistris prefatis quare dictus Archidiaconus ad officium cancellarie nominatus in propria persona non uenit ex quo hoc facere comode potuit saltem ad tam propinqua. Qui ad hoc dicebant quod persona ipsa Episcopo fuerat satis nota. Demum diuerberato negocio hinc et inde et precipue super eo quod dictus nominatus non uenit personaliter: Episcopus tandem finaliter respondit, quod non obstante defectu procuratorii et absencia persone Archidiaconi predicti ea vice de gracia

$$
\text { " Cnis. Arch., Pix. I. } 8 \text {; Rashdall's Universities, ii. } 425 .
$$


speciali dum tamen alias non trahetur ad consequenciam huiusmodi commissio faoienda absenti, ipsos libencius expediret nisi hoc refragaretur, quod prefatus Archidiaconus nominacioni de se facte omnino dissenserat sicut Episcopus asseruit se accepisse pro certo et ideo rogauit vniuersitatem per dictos magistros voce tenus quod debilitati et multiplici occupacioni dicti Archidiaconi compacientes, alium ad dictum officium nominarent, uel si hoc nollent, procurarent consensum dicti Archidiaconi et postmodum mitterent ad eundem Episcopum pro commissione habenda, qui eciam promisit magistris predictis, quod si prefatus Archidiaconus ad eum accederet sicut erat uenturus ad Natale sequens pro consensu adhibendo assumpcioni officii predicti diceret efficaciter quod sentiret. Tandem iij kal. Ianuarii anno predicto apud Tyngehirst venit ex parte vniuersitatis predicte magister Ricardus de Bradele procurator eiusdem petens ab episcopo commissionem concedi et fieri Archidiacono prenotato. Cumque constaret Episcopo per collacionem habitam cum eodem Archidiacono medio tempore prius labente de ipsius dissensu priori mutato in consensum commissionem fieri precepit que facta extitit, et dicto magistro Ricardo magno sigillo signata tradita sub hac forma.

Oliuerus permissione diuina Lincoln. Episcopus dilectis in Christo filiis magistris et scolaribus vniuersitatis Oxon. salutem graciam et benedictionem. Ad instanciam uestre deuocionis officium cancellarie vniuersitatis uestre magistro Simoni de Gandauo Archidiacono Oxon. in theologia inter vos actualiter nunc regenti, ita quod huiusmodi commissio sibi absenti facta ad consequenciam alias non trahetur, ad presens de gracia speciali committimus per presentes donec aliud vobis super hoc dederimus in mandatis. valete. Datum apud Tyngehirst iij kal. Ianuarii Anno domini $\mathrm{M}^{\circ} . \mathrm{CC}^{\circ}$. nonagesimo primo.

Vide plus de hac materia in sequenti folio in parte alba.

[f. $\left.51^{v}\right]$ Deinde viij Id. Ianuarii anno predicto dictus magister Ricardus de Bradele rediens apud Tyngehirst exposuit episcopo qualiter magistri vniuersitatis Oxon. in eundem magistrum Ricardum exscanduerant pro eo quod portauit commissionem prescriptam pro quibusdam nouis uerbis in ea insertis illis scilicet, ita quod huiusmodi commissio facta absenti ad consequenciam alias non trahetur, et idem supplicauit humiliter vt Episcopus sibi confuso quodam modo pie compaciens illa uerba noua de dicta commissione tollere dignaretur presertim cum illa uerba subsequencia, de gracia speciali, pro intencione Episcopi quod non teneatur de necessitate committere absenti sufficienter operarentur ut dicebat. Episcopus uero deliberato aliquantulum cum suis, dicto magistro Ricardo cum tanta humiliacione supplicanti et instanti pro mutacione dictorum uerborum admodum compaciens, illa amoueri precepit concedens com. missionem fieri sub hac forma.

Oliuerus permissione diuina Lincoln. Episcopus dilectis in Christo filiis magistris et scolaribus vniuersitatis Oxon. salutem graciam et benedictionem. Ad instanciam uestre deuocionis officium Cancellarie vniuersitatis uestre magistro Simoni de Gandauo Archidiacono Oxon. in theologia inter uos actualiter nunc regenti ad presens de gracia speciali committimus per presentes donec aliud vobis super hoc dederimus in 
mandatis. valete. Datum apud Tyngehirst viij Id. Ianuarii Anno domini $\mathrm{M}^{\circ} . \mathrm{CC}^{\circ}$. Nonagesimo primo.

Demum dixit Episcopus prefato magistro Ricardo. Nos uidemus qualiter latet anguis in cordibus magistrorum Oxon. et quomodo nituntur reuerti ad antiquam stulticiam suam, et idem dicas eis ex parte nostra et nisi feceris nos faciemus in proximo aduentu nostro ad locum illum si uixerimus, quod tantum fecerunt ista vice quod in proxima nominacione cancellarii ueniet siue dici debeat electus siue nominatus ad nos personaliter, siue simus in propinquo siue in remoto secundum quod-motus animi nostri nobis ingerit in presenti.

Dalderby, Institutions, f. $143^{v}$.

Memorandum quod vj Id. Nouembris anno domini $\mathrm{M}^{\circ} . \mathrm{CCC}^{\circ}$. secundo apud Derteford venerunt ad Episcopum magistri Iohannes de Hale et Willelmus de Anney magistri in artibus apud Oxon. actualiter regentes et exhibuerunt Episcopo quamdam litteram sub hac forma clausam.

Venerabili in Christo patri domino Iohanni dei gracia Lincoln. Episcopo sui si placet deuocionis filii Cetus vnanimis magistrorum vniuersitatis Oxon. obediencie deuocionem patri debite cum omni reuerencia filiali. Debitum pastoralis officii comodis subditorum solerter inuigilans et precipue singularis affeccio pii patris que exuberare dinoscitur circa querentes in studio sciencie margaritas nos excitant et inducunt vt tam in hiis que sunt fauoris et gracie quam consuetudinis seu iusticie consuete ad vestre paternitatis suffragium recurramus intrepide et effectum beniuolum more solito consequamur. Hinc est quod cum Magister Walterus de Wetheringsete sacre theologie professor ad officium cancellarie vacantis per cessionem magistri Iacobi de Cobham iuris canonici professoris per nos nuper vt condecet vnanimiter sit electus beniuolenciam votiuis precibus exoramus paternam quatinus eleccionem ipsius vestra dignetur paternitas absque morose dilacionis obstaculo more solito confirmare, ad quietem studencium paterne consolacionis presidium et laudem ac gloriam creatoris. In prosperitate felici cum mentis leticia vos conseruet ommium retributor. Datum Oxon. viij Id. Nouembris Anno domini $\mathrm{M}^{\circ}$. $\mathrm{CCC}^{\circ}$. secundo.

Et cum sero esset nec vacaret de negocio quod eadem littera tangebat tractare Episcopus dixit eis quod die sequente sequerentur eum vsque ad ciuitatem London. et ibi responderetur eisdem; quo die dictis magistris apud vetus Templum London. coram Episcopo in ipsius camera constitutis et lecta littera memorata quesiuit ab eis Episcopus an alia exhibenda haberent qui protinus exhiberunt quoddam procuratorium et quamdam litteram resignatoriam sub hiis formis.

Venerabili in Christo patri domino Iohanni dei gracia Lincoln. Episcopo deuoti si placet filii Cetus vnanimis magistrorum vniuersitatis Oxon. obediencie debitum cum honore et deuotum reuerencie famulatum. Ad petendum et instancius prosequendum pariter et habendum nostro nomine confirmacionem eleccionis de viro prouido et honesto magistro Waltero de Wetheringsete sacre theologie doctore electo ad Officium cancellarie nuper per nos concorditer inibi celebrate, discretos 


\section{CONFIRMATIONS OF OXFORD CHANCELLORS July}

viros videlicet magistros Iohamnem de Hale procuratorem vniuersitatis predicte et magistrum Willelmum de Anney nostros procuratores legitimos et nuncios speciales ordinamus facinus et constituimus per presentes. Dantes eisdem plenariam potestatem et speciale mandatum petendi et recipiendi nomine nostro confirmacionem eleccionis predicte in personam memorati magistri Walteri ac omnia et singula que dictum eleccionis negocium contingere potuerunt quoquomodo apud vestre paternitatis clemenciam efficaciter prosequendi. Ratum habituri et gratum quicquid per eosdem procuratores seu nuncios actum gestum vel procuratum fuerit in premissis. In testimonium omnis rei sigillum nostrum commune presentibus duximus apponendum. Floreat vestre paternitatis benignitas diu in prosperis et secundis fructificet incrementis. Datum Oxon. viij Id. Nouembris Anno domini $\mathrm{M}^{\circ}$. $\mathrm{CCC}^{\circ}$. secundo.

Venerabili in Christo patri ac domino Reuerendo domino I. dei gracia Lincoln. Episcopo suus humilis et deciotus Iacobus de Cobeham reuerenciam debitam cum honore. Quia plurimis ac variis negociis prepeditus existo ita quod ad officium Cancellarii Oxon. gerendum seu etiam exercendum ad presens venire non valeo, de vestre reuerende paternitatis licencia dicto officio cedo per presentes. In cuius rei testimonium sigillum meum presentibus est appensum Anno domini $\mathrm{M}^{\circ} \cdot \mathrm{CCC}^{\circ}$. secundo penultimo die mensis Octobris.

Deinde examinato registro Episcopi et predecessorum suorum super processibus habitis cum nominatis ad officium cancellarie vniuersitatis Oxon. temporibus retroactis inuentum fuit quod forma dicte littere vniuersitatis Episcopo misse per quam petebatur electus magistrorum eiusdem more solito confirmari ab aliis litteris in casu huiusmodi prius missis in forma totaliter discrepare. Et episcopo querente a magistris predictis quare forma fuit commutata, iidem magistri responderunt quod solum fuit mutata pro eo quod forma antiqua non videbatur satis competens et ornata tanto domino destinanda: deinde quesitum fuit ab eisdem quare nominatus non venit personaliter sicut tenetur et facere consueuit, et responderunt quod si venisset cessatum fuisset in ipsius absencia a lectura per alios magistros professionis sue et oportuit ipsum magnas fecisse expensas in eundo et redeundo precipue Episcopo extra diocesim existente quarum dicta vniversitas penuriam paciebatur vt dixerunt. Et episcopus ad hoc, quando non fuit nisi vna cista communis que rocatur Lincoln. in vniuersitate predicta tune habuit vniuersitas sumptus pro negociis suis expediendis et nuno tales ciste multiplicate ex quibus potuissent dicti magistri copiam expensarum saltem ex mutuo habuisse, et dictis [f. 144] magistris respondentibus se ab vniuersitate predicta nihil recepisse nomine expensarum, quesitum fuit $a b$ eis quare maiores rniuersitatis ad episcopum non venerunt sicut fieri consuenit siue nominatus veniret personaliter siue vniuersitas mitteret pro commissione sibi absenti de gracia facienda, et ipsi allegarunt expensarum penuriam sicut prius: super quibus habita deliberacione, episcopus respondit eisdem quod citra Natale ad aliquod manerium ecclesie sue a municipio $O x o n$ per septem miliaria ad minus concedente domino declinaret et ibi posset nominatus ad eum venire personaliter absque carencia leccionum et grauamine expensarum, sibique taliter venienti paratus foret 
facere quicquid iuris esset in casu huiusmodi siue moris. Fit ne pro defectu commissionis huiusmodi dampnum vel periculum in vniuersitate predicta interim imineret: optulit eis Episcopus de gracia commissionem dicto nominato faciendam vsque ad festum Natalis predictum. Sed dicti magistri asserentes se talem commissionem temporalem recipere non audere, protinus recesserunt. Postmodum xv kal. Decembris anno predicto episcopo apud Tinghirst existente venerunt ad ipsum ibidem magistri Ricardus fuge in iure canonico, Dyonisius Auenel in iure ciuili, Oliuerus Deyncurt et Iohannes de sancto Amando in artibus actualiter regentes in vniuersitate pretacta et exhibuerunt Episcopo litteram eiusdem vniuersitatis sub continencia infrascripta.

Reuerendo patri suo in Christo et domino Iohanni dei gracia Lincoln. Episcopo vniuersitatis Oxon. cetus humilis magistrorum salutem et obedienciam debitam et deuotam. Affeccionis paterne dileccionem habere vos condecet circa querentes in agro studii sciencie margaritam que domum dei multipliciter conuenustat in hiis que precipue non possunt absque denegacione gracie ac iuris iniuria denegari. Hinc est quod cum magister Walterus de Wetheringsete sacre theologie professor a nostra vniuersitate ad officium Cancellarie per cessionem magistri Iacobi de Cobham vacantis concorditer sit electus a vestre paternitatis beniuolencia eius eleccionem more solito petimus confirmari, talem ergo si placet vos exhibeatis in hac parte vt filiorum vestrorum sinceritas augeatur et paterne dileccionis caritas comprobetur: valeat paternitas vestra reuerenda per tempora longiora. Datum Oxon. xvij kal. Decembris Anno domini $\mathrm{M}^{\circ}$.CCC ${ }^{\circ}$. secundo.

Asseruerunt requisiti se nihil aliud exhibendum habere sed petebant quod Episcopus eleccionem factam de dicto magistro Waltero more solito confirmaret. Et cum diceretur eis quod non fuit eleccio sed simplex nominacio reputanda, nec confirmari sed officium Cancellarie nominato per vniuersitatem vsque ad beneplacitum Episcopi committi solummodo consueuit: petebant quod siue esset confirmacio siue commissio facienda, episcopus eos more solito expediret. Habitaque altercacione diutina super eo quod nominatus personaliter non venit, tandem dixit Episcopus, aut petitis quod huiusmodi negocium in absencia nominati expediatur de iure et consuetudine vel etiam graciose, et illis respondentibus se nihil velle petere nisi quod episcopus huiusmodi negocium more solito siue de iure vel consuetudine siue de gracia pro suo arbitrio ${ }^{1}$ expediret, dixit Episcopus quod gracia nisi petenti facienda non foret, vnum iniunxit eis quod deliberarent vsque in crastinum et sibi super disiunctiua huiusmodi responderent. In crastino vero dicti magistri coram episcopo personaliter constituti vt prius peticioni pristine institerunt asserentes se mandatum petendi aliud non habere. Et quia in sua peticione se semper ad morem solitum referebant, recitatum fuit eis registrum de commissionibus factis magistris Iacobo de Cobham, Ricardo de Clyue, Rogero de Weseham et Rogero de martiuall factis in absencia eorundem in quo continebatur quod singulis vicibus commissionem huiusmodi fieri de gracia petebatur. Quo viso dicti magistri dicentes se velle super huiusmodi peticione cum vniuersitate

1 libito deleted. 
deliberare, licenciati ab episcopo recesserunt. Postea xij kal. Ianuarii anno predicto apud Thame vbi episcopus adtunc fuit memorato magistro Waltero de Wetheringsete in Cancellarium nominato ibidem personaliter existente venerunt magistri Dionisius Auenel predictus Iohannes procurator vniuersitatis Oliuerus Deyncourt, Iohannes de sancto Amando ${ }^{2}$ et prefatum magistrum Walterum per vniuersitatem in cancellarium vt premittitur nominatum eidem Episcopo presentarunt humiliter postulantes quod idem nominatus personaliter comparens expediretur more solito circa personaliter comparentes primitus obseruato: dicto nominato obiecit quod de officio Cancellarie se intromiserat eodem officio vacante in preiudicium ipsius Episcopi a quo idem est officium affirmandum ${ }^{3}$; ad quod dictus nominatus respondit se nihil exercuisse de hiis que spectant ad iurisdiccionem spiritualium sed de hiis que contingunt regiam potestatem, et episcopus adiecit, quod nec vnum nec aliud antequam esset cancellarius debuerat attemptasse, exemplum ponens in episcopis et aliis prelatis regalia optinentibus qui licet ad episcopatum vel aliam prelatinam eligantur et a Rege admittantur de huiusmodi regalibus se non possunt intromittere donec per superiorem suum ecclesiasticum eleccio confirmetur. Super quibus habita altercacione aliquali episcopus huiusmodi culpam dicto nominato ea vice remittens eidem commissionem tradidit sub hac forma.

Iohannes permissione diuina Lincoln. Episcopus dilectis in Christo filis magistris et scolaribus vniuersitatis Oxon. vniuersis salutem graciam et benediccionem. Ad instanciam vestre deuocionis officium cancellarie vniuersitatis vestre magistro Waltero de Wetheringsete in theologia inter vos actualiter nunc regenti per vos nobis ad idem officium nominato et ad nos propter hoc apud Thame personaliter venienti et coram nobis presencialiter constituto duximus committendum donec aliud vobis super hoc dederimus in mandatis. Datum apud Thame xiij kal. Ianuarii Anno domini $\mathrm{M}^{\circ}$.CCC $\mathrm{CC}^{\circ}$ secundo et Consecracionis nostre Tertio.

Becke, Institutions, f. $9 \tilde{5}$.

Memorandum quod vj Idus Marcii Anno domini Millesimo C'CCmo quadragesimo sexto apud Lincoln. Magister Thomas de Buktone iuris ciuilis professor et Rogerus Lok officium procuratoris vniuersitatis Oxon. gerens coram Officiali Lincoln. sede vacante personaliter constituti exhibuerunt. eidem litteram vniuersitatis predicte clausam sub hac forma.

Reuerende discrecionis viro domino Custodi spiritualitatis Episcopatus Lincoln. sede vacante, eiusve locum tenenti vniuersitatis Oxon. cetus humilis Magistrorum obedienciam debitam ac deuotam. Affeccionis sincere dileccionem habere vos condecet erga querentes in agro studii sciencie margaritam que domum dei multipliciter conuenustat in hiis que precipue non possunt absque denegacione gracie ac iuris iniuria denegari, hinc est quod Magister Iohannes de Northwode doctor sacre theologie a nostra vniuersitate ad officium cancellarie iterato concorditer est electus; a vestra reuerenda beniuolencia, eius eleccionem more solito

$$
\text { = ISS. Durando. = MS. affirmendum. }
$$


petimus confirmari, talem erga vos si placet exhibeatis in hac parte vt deuotorum vestrorum sinceritas augeatur et benigne dileccionis caritas comprobetur. Valeat dominacio vestra reuerenda per tempora longiora. Datum Oxon. ij die Mensis Marcii Anno domini Millesimo CCCmo slvjto.

Eisdem die et loco exhibuerunt insuper prefati Magistri Thomas et Rogerus eidem Officiali quoddam procuratorium patens sub hac forma.

Reuerende discrecionis viro domino Custodi spiritualitatis Episcopatus Lincoln. sede vacante eiusve locum tenenti vniuersitatis Oxon. Cetus humilis magistrorum salutem et obedienciam debitam ac deuotam. Nouerit discrecio vestra reuerenda, quam in singulorum nostrorum negociorum promocione semper graciosam recepimus et beninolam, quod nos dilectos nobis in Christo conmagistros nostros et amicos Magistrum Thomam de Buktone iuris ciuilis professorem et Magistrum Rogerum Lok officium procuratoris nostre vniuersitatis gerentem nostros veros et legitimos procuratores et nuncios speciales et quemlibet eorum insolidum coniunctim et diuisim constituimus et facimus per presentes ad petendam et recipiendam confirmacionem et commissionem more solito a vestra dominacione reuerenda electi nostri in cancellarium: ratum habituri et gratum quicquid dicti procuratores vel nuncii nostri vel quilibet eorum in premissis duxerint vel duxerit faciendum. In cuius rei testimonium sigillum commune vniuersitatis nostre predicte presentibus duximus apponendum. Datum Oxon. dicto die Mensis Marcii Anno domini Millesimo CCCmo xlvjto.

Quibus perlectis statim Officialis quesiuit ab eisdem procuratoribus quid petere vellent, ipsi protinus respondebant, petimus, domine, Electum nostrum in cancellarium more solito expediri, quibus Officialis respondit, illum quem vos nominatis Electum, nos vocamus nominatum, et quare non venit personaliter sicut et alii eum precedentes facere consueuerunt; qui dixerunt quod tunc propter pericula que in absencia cancellarii inter scolares ad vniuersitatem Oxon. vndique confluentes poterunt de facili iminere, se nequiuit aliqualiter absentare, et ideo absenti huiusmodi commissionem fieri more solito instancius postularunt. Et cum quereretur per Officialem ab eis vtrum de iure vel de gracia hoc fieri petebant, dicebant, Domine reuerende, nos petimus a discrecione vestra reuerenda iuxta formam solitam hac vice graciose expediri. Et statim Officialis respondit sub hiis verbis. Quia vos petitis graciam, ideo vobis graciam specialem facimus ista vice et precepit eis fieri quandam commissionem sub hac forma.

Willelmus Bachiler canonicus ecclesie Lincoln. Officialis Lincoln. sede vacante dilectis nobis Magistris et scolaribus vniuersitatis Oxon. Lincoln. diocesis salutem in amplexibus saluatoris. Ad instanciam vestre deuocionis officium cancellarii vniuersitatis predicte Magistro Iohanni de Northwode sacre pagine professori inter vos actualiter nunc regenti quamuis absenti ob certas causas coram nobis allegatas et sufficienter probatas hac vice de gracia speciali committimus per presentes quousque nos seu alius iure episcopali Lincoln. diocesis presidens aliud vobis super hoc dederimus seu dederit in mandatis. Datum apud 
Lincoln. vjto Id. Marcii Anno domini Millesimo CCCmo quadragesimo sexto.

Chancellor elected

John de Monemuta

Simon de Gandavo

Roger de Martivall

Roger de Wesenham

Richard de Clive

James de Cobeham

Walter de Wetheringsete

Simon de Favresham

Walter Burdun

William de Bosco

Henry de Mamesfeld

Henry de Harkele

John Lutterel

Henry de Gower

William de Alberwyk

Thomas de Hothom

Roger de Stretton

Nigel de Wauere

Richard FitzRalph (Ricardus Radulfi)

Hugh de Willughby

Robert de Stretteford

John de Lecche

William de Skelton

William de Bergeveney

William de Bergeveny

John de Northwode

John de Northwode

John de Echingham

William Courtenay
Retiring Chancellor

Villiam de Kingscote

per cess. J. de Monemuta

per resig. S. de Gandavo

per cess.

R. de Martivall

,, R. de Wesenham

, R. de Clyve

., J. de Cobham

, IV. de Wetheringsete

, S. de Farresham

"W. de Bordon

per renun. W. de Bosco

per cess. H. de Mammesfeld

per mort. H. de Haircla

per revoc. J. Lutterel

per cess. H. de Gouwer

, W. de Alburwyk

, Ralph de Salopia

, R. de Stretton

N. de Wauere

per cess. R. FitzRalph

, H. de Wiloughby

Office vacant

per cess. J. de Lecche

, W. de Skelton

, Re-elected

, W. de Bergeveny

Re-elected

per cess. John de Renham

Adam de Toneworth
Date of confirmation

6 June $1290^{1}$

30 Dec. $1291^{2}$

6 Jan. 1292

22 Nov. $1293^{3}$

$\{23$ Nov. 1293

11 Feb. 12954

2 Nov. $1297^{\circ}$

8 May $1300^{\circ}$

20 Dec. $1302^{\circ}$

31 Jan. $1304^{F}$

14 Feb. $1306^{\text {s }}$

6 Apr. 1308 "

27 Oct. 1309"

11 Dec. 1312 i:

15 Oct. $1317^{13}$

18 Oct. 1322 :-

3 Nov. $1325^{1:}$

25 Jan. 1327

12 Nov. 1329 17

30 Nov. $1330^{\text {Is }}$

30 May 1332 's

14 May $1334^{20}$

9 May $1335^{\approx 1}$

10 Nov. $1338^{2:}$

26 Dec. 1339 ="

10 June $1341^{23}$

10 June 1343 :

15 May $1345^{2+\mathrm{i}}$

10 Mar. $1347^{-3}$

15 June $1363^{2 *}$

$10 \mathrm{June} 1367^{\mathrm{gs}}$

\section{A Defence of the Proscription of the Yorkists in 1459}

Attention has never, I think, been called to the subjoined fragment. Though unfortunately mutilated at the beginning, and in places corrupted by a bad copyist, it probably contains all the important part of a curious political pamphlet. The date is clearly the latter part of 1459 or beginning of 1460 , and the aim seems to be to prevent any weakening on Henry VI's part in the

- Sutton, Mem.f. $3 . \quad=$ Ibid. f. 50 .

3 Ibid. f, $86^{\circ}$.

+ Ibid. f. $11 \%$.

5 Jbid. f. 178.

"Dalderby; Mem. f. $5 \mathrm{r}$. ' Dalderby, Instit. f. 143'.

M Ibid. f. 145.

I Ibid. f. 149.

10 Jbid. f. 153.

11 Ibid. f. $154^{2}$.

$1=$ Ibid. f. 161 .

13 Ibid. f. 168.

it Burghersh, Instit. f. $\mathbf{2 4 8}$.

is Ibid. f, $252^{\mathrm{v}}$.

16 Ibid. f. $258^{*}$

17 Ibid. f. 261 .

I Ibid. f. 203 .

1. Ibid. f. $266^{\vee}$ (Ricardus Radi in the MS., incorrectly transcribed by llood and in Munimenta Academica as Ricardus Radin).
$=0$ Ibid. f. $268^{\circ}$.
$\because$ Ibid. f. 272 .
$\because \quad$ Ibid. f. $280^{\circ}$.
23 Ibid. f. $2 \widehat{3} 3$.
$\because$ Ibid. f. 288v. $\approx$ Becke, Instit. f. $S 8^{v}$.
: Ibid. f. 927.
$=$ Ibid. f. 9.5.
:* Buckingham, Instit. f. 335 .
Io Ibid. f. $341^{5}$. 